\title{
A COMPARATIVE STUDY ON THE TWO VIBRATION DRIVEN LOCOMOTION SYSTEMS IN VARIOUS FRICTION LEVELS
}

\author{
Ngoc-Tuan La ${ }^{1}$, Thanh-Toan Nguyen ${ }^{2}$, Van-Du Nguyen ${ }^{3, *}$ \\ ${ }^{1}$ Faculty of Manufacturing, Vinh University of Technology Education, Vietnam \\ ${ }^{2}$ Center of Information and Library service, Thai Nguyen University of Technology, \\ Thai Nguyen University, Vietnam \\ ${ }^{3}$ Faculty of Mechanical Engineering, Thai Nguyen University of Technology, \\ Thai Nguyen University, Vietnam \\ *E-mail: vandu@tnut.edu.vn
}

Received: 04 November 2020 / Published online: 21 April 2021

\begin{abstract}
This paper presented comparison results of two locomotion models: a purevibration driven and a vibro-impact driven system. In experiments, the friction force can be varied without changing the internal and the body masses. The mathematical models of the two systems were developed and experimentally verified. Using dimensionless models, the results can be expanded to other sizes in practice. The two models were compared in the following aspects: the progression rate, the motion direction and the dynamics response. The effect of friction as an important variable on the dynamic response of the two scaled models were examined and compared by means of bifurcation analysis and basin of attraction. It has been found that, the pure-vibration can provide forward motion better than the vibro-impact does. The highest progression rate of the vibro-impact was less than that of the pure-vibration system in the investigated ranges of input parameters. Besides, the pure-vibration always has period-1 motion, whereas the vibro-impact system has a rich and complex dynamic response, including period-1, period-2 as well as chaotic motions. The results obtained would be useful for design and operating the self-propelled locomotion systems.
\end{abstract}

Keywords: self-propelled locomotion; vibration-driven; vibro-impact; bifurcation; basin of attraction.

\section{INTRODUCTION}

Recently, there has been remarkable development of mobile systems, especially in the field of locomotion robots. The systems have potential applications, either in industrial remote control robots [1-3] for remote and hostile environments, disaster rescues, pipeline inspection ... [4] or in micro-size robots for medical assistance [5]. Conventional mobile robots usually consist of external impulsion components (wheels, paddles, legs). 
Such structure would lead to several disadvantages, for example, the mechanical complexity, difficult to control, large dimensions, failure of moving components, or would make damage to the surrounding environment. Consequently, mobile devices driven by vibration have been recently developed, providing a promising solution for the above mentioned problems. The vibration driven locomotion system was initially proposed by Chernous'ko [6], based a principle where the rectilinear motion can be achieved by the interaction force between the internal mass moving inside the body frame. Such relative motion of the two massed must follow a specially designed multi-phase accelerationcontrolled [7-10]. A number of driving actuators have been proposed to provide the relative motion of the internal mass inside the body, such as pendulum with DC motor [11], eccentric rotary mass [12,13], electromagnetic device [7-10]. In general, one of the biggest shortcomings of the vibration driven locomotion is that it requires a complex control strategy. A new solution was recently found [14] where the locomotion is caused without impact but not requires a complex motion control algorithm.

Another design principle for self-locomotion system is based on the vibration combining impact force. Such system are well known as vibro-impact locomotion system. In this system, the internal mass oscillates and periodically hits the body, leading to movement of the whole system. This principle was first time proposed and analyzed by Pavlovskaia et al. [15]. Many studies have been made, using various types of the actuators, such as using a solenoid driven by an RLC circuit $[16,17]$, electromagnetic device [18,19], linear motors [20], electro-dynamic shaker [21,22]. The expected direction of the system motion can be obtained by applying a position feedback controller [23] or reversing the impact side [24]. A rich and complex dynamic response of the vibroimpact system was confirmed, as a result of the co-existence of non-smooth friction and the impact force $[25,26]$. The system response has been found to be either periodic, quasiperiodic and chaotic response, and thus required a careful design [21] and/or parameter selection [27] to make the system work stable. However, experimental studies on the effects of the environment friction as well as the interaction between the friction and the excitation force on the system response have rarely been found $[14,16]$. Moreover, some exciting notes have been marked for such systems [20-23]. For example, when the elastic force exerting on the system is greater than the threshold of the friction, the system may have backward motion [23]. It has been revealed that the performance of the capsule system may not be improved by simply applying a large amplitude of excitation [20]. Nevertheless, these issues were only found at a fixed value of friction force in experimental studies.

Consequently, this study was made to compare the two systems: pure-vibration locomotion and vibro-impact locomotion, regarding their ability to move forward and their dynamic response under various levels of friction force.

The paper is organized as below. Firstly, the mathematical models describing the two systems are briefly presented in Section 2. The experimental setup and experimental operations used to validate the models are then described in Section 3. Section 4 shows major analyses to compare the two systems, regarding their progression velocity (both direction and magnitude) and their dynamical response. Some important remarks and recommendations are made in the conclusion section. 


\section{MATHEMATICAL MODELING}

The physical model of a representative vibro-impact propulsion system is shown in Fig. 1(a). The system contains a body mass, $m_{2}$ and an inertial mass, $m_{1}$. A nonlinear spring $k$ and a viscous damping, $c$ connecting the two masses. The body mass $m_{2}$ can move along a straight line on a resistive horizontal direction. The internal mass was excited so as to oscillate in the same direction with the motion of the system. A sinusoidal force $F_{m}$ with amplitude $A$ and frequency $\Omega$ acts on the two masses. The friction force, $F_{r}$ between the body mass and the environment is assumed to follow the Coulomb-Stribeck frictional model. When the force exerting on the body greater than the threshold of the static value of the friction $F_{s}$, a bidirectional motion of the system can be generated.

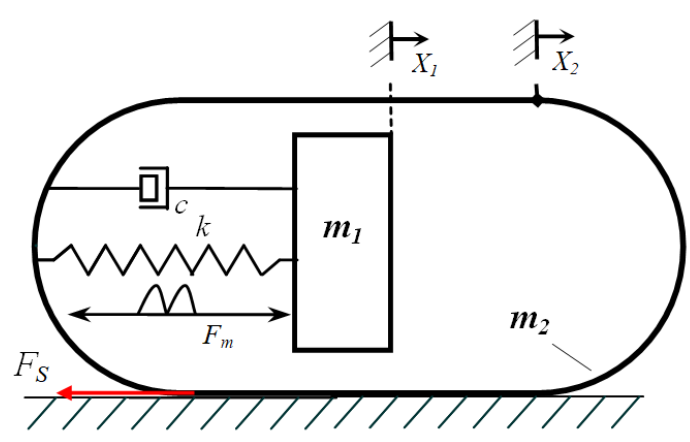

(a) Pure-vibration

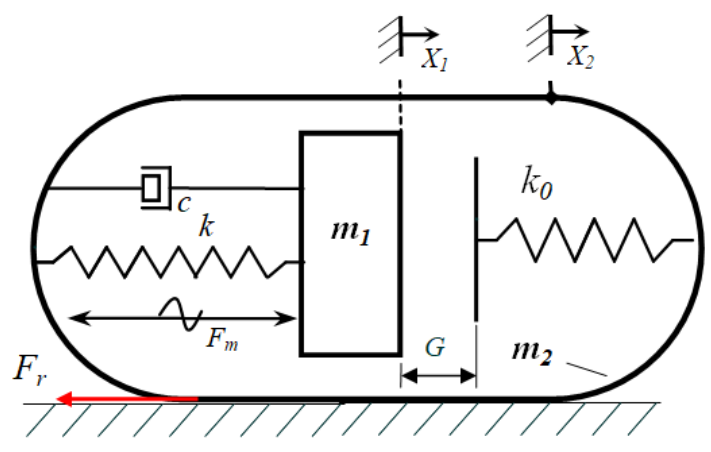

(b) Vibro-impact

Fig. 1. The model of the self-propelled systems: (a) Pure-vibration and (b) vibro-impact driven systems

In Fig. $1, X_{1}$ and $X_{2}$ denote the absolute displacements of the two masses, $m_{1}$ and $m_{2}$, respectively. A forward movement is assigned for positive values of $X_{i}(i=1,2)$, and vice versa. For the pure-vibration system (Fig. 1(a)), the internal mass is driven by a halfsine excitation and does not have any impact with the body (see [14] for more details). For the vibro-impact system (Fig. 1(b)), the two masses have a gap $G$ at the balance position. The impact force generated when the relative displacement $X_{1}-X_{2}$ is greater than or equal to the gap $G$ would result in the motion of the system. The impact stiffness was modeled as $k_{0}$.

Since most real springs exhibit a nonlinear force-deflection relation, the spring force was defined as a function of distance between of the two masses in a cubic form, as similar to ones in $[14,21]$ as following

$$
F_{s p r}=k_{1}\left(X_{1}-X_{2}\right)+k_{2}\left(X_{1}-X_{2}\right)^{3} .
$$

The friction force was found to have an important role for movement of the vibrationdriven locomotion systems $[6,15]$. It has been revealed that the average velocity of the system progression can be increased if the friction between the body and environment is increased and the friction between the two masses is reduced [6]. However, it was 
also found that the system may not have forward motion or even move backward if the friction force is too small or enormous.

The operation of the system can be analyzed to follow two stages. The first stage is the one where the mass $m_{1}$ is not in contact with the mass $m_{2}$, i.e. $\left|X_{1}-X_{2}\right|<G$.

$$
\left\{\begin{array}{l}
m_{1} \frac{d^{2} X_{1}}{d t^{2}}=F_{e}-F_{s p r}-c\left(\frac{d X_{1}}{d t}-\frac{d X_{2}}{d t}\right) \\
m_{2} \frac{d^{2} X_{2}}{d t^{2}}=-F_{e}+F_{s p r}+c\left(\frac{d X_{1}}{d t}-\frac{d X_{2}}{d t}\right)-F_{r}
\end{array}\right.
$$

This equation is also used as the mathematical model of the pure-vibration driven system.

The second stage happens when the relative displacement of the two masses is equal to or larger than the gap, i.e. $X_{1}-X_{2} \geq G$. As this instant, the internal mass $m_{1}$ impacts with the body. The equations describing the system at this stage can be expressed as

$$
\left\{\begin{array}{l}
m_{1} \frac{d^{2} X_{1}}{d t^{2}}=F_{e}-F_{s p r}-c\left(\frac{d X_{1}}{d t}-\frac{d X_{2}}{d t}\right)-k_{0}\left(X_{1}-X_{2}-G\right) \\
m_{2} \frac{d^{2} X_{2}}{d t^{2}}=-F_{e}+F_{s p r}+c\left(\frac{d X_{1}}{d t}-\frac{d X_{2}}{d t}\right)+k_{0}\left(X_{1}-X_{2}-G\right)-F_{r}
\end{array}\right.
$$

A complete equation of the vibro-impact system, combining the two above stages, is then expressed by combining Eqs. (2) and (3) as

$$
\left\{\begin{array}{l}
m_{1} \frac{d^{2} X_{1}}{d t^{2}}=F_{e}-F_{s p r}-c\left(\frac{d X_{1}}{d t}-\frac{d X_{2}}{d t}\right)-H k_{0}\left(X_{1}-X_{2}-G\right) \\
m_{2} \frac{d^{2} X_{2}}{d t^{2}}=-F_{e}+F_{s p r}+c\left(\frac{d X_{1}}{d t}-\frac{d X_{2}}{d t}\right)+H k_{0}\left(X_{1}-X_{2}-G\right)-F_{r}
\end{array}\right.
$$

where $H($.$) is the Heaviside function defined as$

$$
\left\{\begin{array}{l}
H=1, X_{1}-X_{2}-G>0 \\
H=0, X_{1}-X_{2}-G \leq 0
\end{array}\right.
$$

For the pure-vibration driven system, the mathematical model is described by Eq. (2), with a half-sine excitation force, which was defined as

$$
\begin{cases}F_{e}=\sin (\Omega t), & \sin (\Omega t)>0 \\ F_{e}=0, & \sin (\Omega t) \leq 0\end{cases}
$$

For the vibro-impact driven system, the excitation force was set in the form of fullsine wave, i.e.

$$
F_{e}=A \sin (\Omega t)
$$

When studying the dynamics response of mechanical systems, dimensionless models have usually been developed. It has been found that the variables and parameters in 
Eq. (3) were transformed as following (see [24,27-29] for example)

$$
\begin{aligned}
& \tau=\Omega_{0} t ; x_{1}=\frac{k_{1}}{F_{r}} X_{1} ; x_{2}=\frac{k_{1}}{F_{r}} X_{2} ; \Omega_{0}=\sqrt{\frac{k_{1}}{m_{1}}} ; \omega=\frac{\Omega}{\Omega_{0}}, \\
& \zeta=\frac{c}{2 m_{1} \Omega_{0}} ; \alpha=\frac{A}{F_{r}} ; \sigma=\frac{k_{0}}{k_{1}} ; \beta=\frac{k_{2}}{k_{1}}\left(\frac{F_{f}}{k_{1}}\right)^{2} ; \gamma=\frac{k_{1}}{F_{r}} G ; \mu=\frac{m_{2}}{m_{1}} .
\end{aligned}
$$

And the dimensionless equations of the vibro-impact driven system were defined as

$$
\left\{\begin{array}{l}
\frac{d^{2} x_{1}}{d \tau^{2}}=\alpha \sin (\omega \tau)-\left(x_{1}-x_{2}\right)-\beta\left(x_{1}-x_{2}\right)^{3}-2 \zeta\left(\frac{d x_{1}}{d \tau}-\frac{d x_{2}}{d \tau}\right)-h \sigma\left(x_{1}-x_{2}-\gamma\right) \\
\frac{d^{2} x_{2}}{d \tau^{2}}=\left[-\alpha \sin (\omega \tau)+\left(x_{1}-x_{2}\right)+\beta\left(x_{1}-x_{2}\right)^{3}+2 \zeta\left(\frac{d x_{1}}{d \tau}-\frac{d x_{2}}{d \tau}\right)+h \sigma\left(x_{1}-x_{2}-\gamma\right)-\operatorname{sgn}\left(\frac{d x_{2}}{d \tau}\right)\right] \frac{1}{\mu}
\end{array}\right.
$$

where $h$ is the Heaviside function for impact conditions in dimensionless form

$$
\left\{\begin{array}{l}
h=1, x_{1}-x_{2}-\gamma \geq 0 \\
h=0, x_{1}-x_{2}-\gamma<0
\end{array}\right.
$$

In above mentioned studies, the effect of the excitation force $\left(F_{m}\right)$ was examined by using the force ratio, $\alpha$. As above mentioned, the friction force was usually kept as a constant. Consequently, different excitation levels can be reflected by various values of the force ratio, $\alpha$. However, it would not be convenient to use such force ratio to reflect the variation of the friction force. For example, a value of $\alpha=2$ may refer any couples of $\left(A=2, F_{r}=1\right) ;\left(A=1 ; F_{r}=0.5\right)$ etc. Our experimental results found that, applying the same force ratio $\alpha$ and varying the friction force would result in either forward or backward motion of the system.

In this study, the mathematical models (2) and (4), corresponding to pure-vibration and vibro-impact driven locomotion systems, respectively, will be validated by experimental data. The verified models will then normalized into dimensionless forms. The effect of the friction force as a variable on the two systems then compared, regarding to their motion direction and dynamical response, by using numerical solution of the normalized models with the same input parameters.

\section{EXPERIMENT SETUP AND VALIDATION}

\subsection{Experiment setup}

The experimental setup was implemented as shown in Fig. 2. A mini shaker (1) is fixed on a linear bearing guide (4), providing a very small friction force. A carbon tube (6) having very small weight, able to slide inside two aluminium V-block (7), was connected with the shaker body. The two V-blocks are combined with two electromagnets, providing a controlled clamping force and thus an adjustable sliding friction. The total friction including two terms: the tiny component, generated by the linear bearing, and the larger component, as a result of the clamping force. This configuration providing an ability of varying the friction force while the body mass was kept constant. Using this setup, the system behavior in different friction magnitudes can be easily examined. 


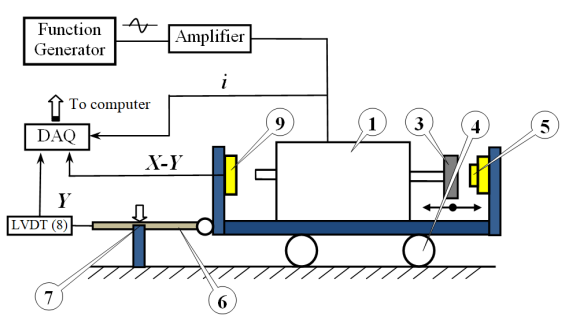

(a)

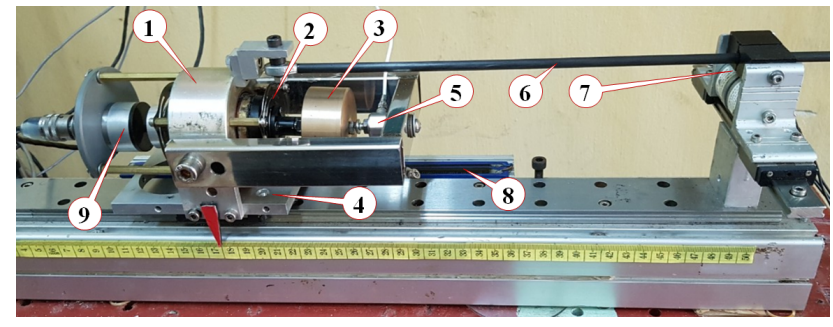

(b)

Fig. 2. (a) The experimental schema and (b) a photograph of the experiment apparatus

Generally, the shaker is excited by applying a sinusoidal current, resulting in a relative motion of the shaker shaft. An obstacle block (5) was placed on the oscillation path of the shaker shaft, with a preset gap $G=0.5 \mathrm{~mm}$. The internal mass, $m_{1}$, is practically include the addition mass and the shaker shaft. A non-contact position sensor (9) was used to measure the relative displacement of the two masses. The absolute displacement of the system was captured by a linear variable displacement transformer (LVDT). The body mass $m_{2}$ consists of the shaker body, the sensors attached on and the carbon tube. The signals from the sensors were captured by a data acquisition system (DAQ) and then stored and analyzed.

\subsection{Experiment operations}

The excitation force was controlled by adjusting the supplied current. An additional experiment was implemented to carry out the relationship between the magnetic force generated between the two massed and the current supplied. A load cell was used to fix the shaker. A DC voltage was supplied to the shaker to generate the magnetic force. Varying the voltage, several pairs of the current passing the shaker and the force measured by the load cell were collected. Our experimental data discovered that the force is linearly dependent to the current applied (see [21] for details).

A sinusoidal signal was generated by mean of the signal generator of a commercial USB PC oscilloscope, model 2204A. The signal was then enlarged by a stock-available power amplifier and supply to the shaker. The current applying to the shaker can be adjusted by changing the signal amplitude from the driving software of the oscilloscope. For vibration driven without impact experiments, the shaker was driven by a half-sine powered current, obtained using a current rectifier.

\subsection{Experimental validation}

For no-impact vibration-driven locomotion system, the model (2) was validated for several sets of experiments. Detailed results can be found in [14]. Fig. 3 illustrates two time histories compared between numerical solution of Eq. (2) and experimental data obtained with the same parameters.

As can be seen from the figure, there was a close similarity between simulation and experimental results. The same progression rate can be observed on both simulation and experimental plots. It is also seen that on both simulation (Fig. 3(a)) and experimental 
data (Fig. 3(b)), the internal mass oscillation has a small-amplitude cycle between two larger-amplitude cycles. The frequency of coupled large- and small-amplitude cycles is exactly the frequency of the excitation force.

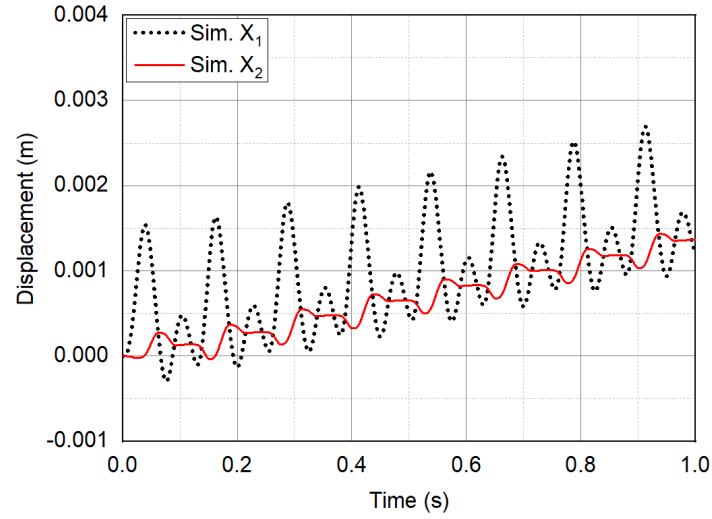

(a)

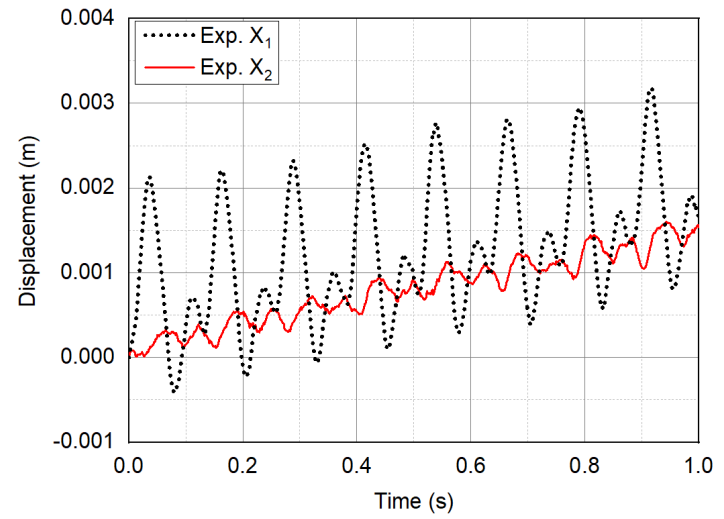

(b)

Fig. 3. Time history of the motions of the internal mass $\left(X_{1}\right.$, dotted line) and of the body $\left(X_{2}\right.$, solid line) from (a) simulation results and (b) experimental data; an excitation frequency of $8 \mathrm{~Hz}$ and the friction force of $2.02 \mathrm{~N}$ were applied

Fig. 4 shows another validation of the model for the case of backward motion. There also appeared a similar between numerical solution and experimental data, in terms of both motion form and progression rate.

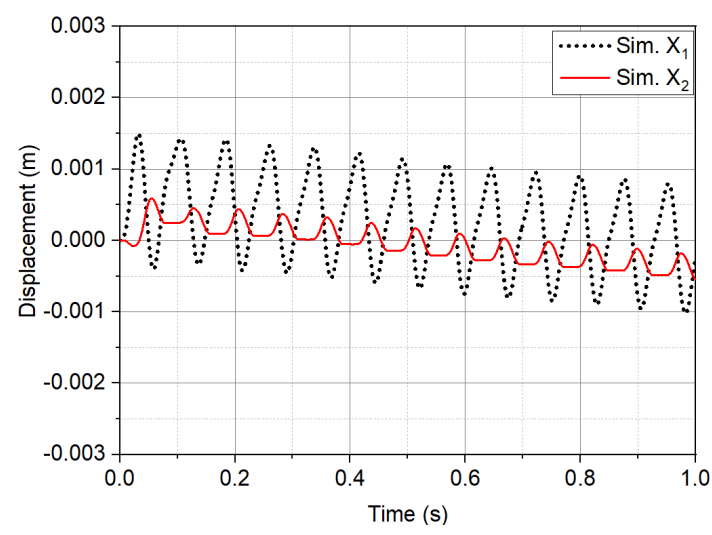

(a)

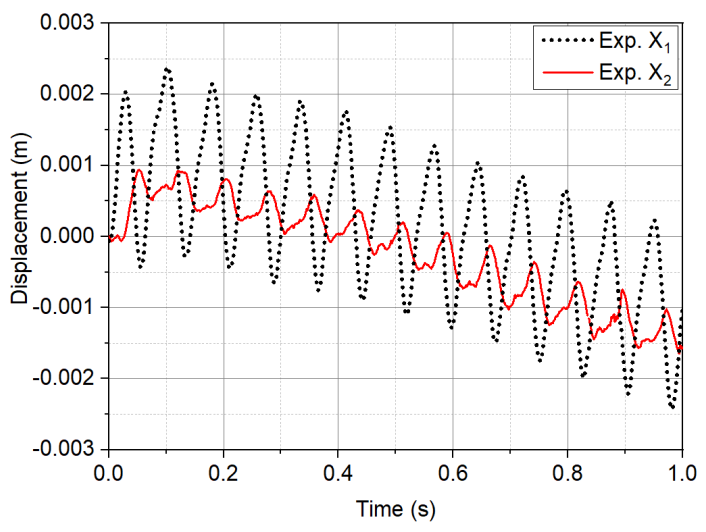

(b)

Fig. 4. Time history of the motions of the internal mass $\left(X_{1}\right.$, dotted line $)$ and of the body $\left(X_{2}\right.$, solid line) from (a) simulation results and (b) experimental data; An excitation frequency of $13 \mathrm{~Hz}$ and friction force of $2.02 \mathrm{~N}$ were applied 
In order to validate the mathematical model for vibro-impact system (Eq. (4)), the numerical solution and experimental data were compared. Fig. 5 illustrates two comparative examples of forward and backward motions. The time histories were built for two levels of friction force: the low friction, $F_{S}=2.3 \mathrm{~N}$ (Fig. 5(a) and 5(b)) and the high friction, $F_{S}=13.6 \mathrm{~N}$ (Figs. 5(c) and 5(d)). Figs. 5(a) and 5(c) show the simulation results of Eq. (4), whereas Figs. 5(b) and 5(d) show the corresponding plots of the experimental data. Overall, it can be observed a good agreement between the simulation progression rate and those from experiments. A slightly different between the experimental trajectories and those from numerical solution appeared on the plots. That would due to the several errors in modelling or experimental measurements of the elastic force. However,

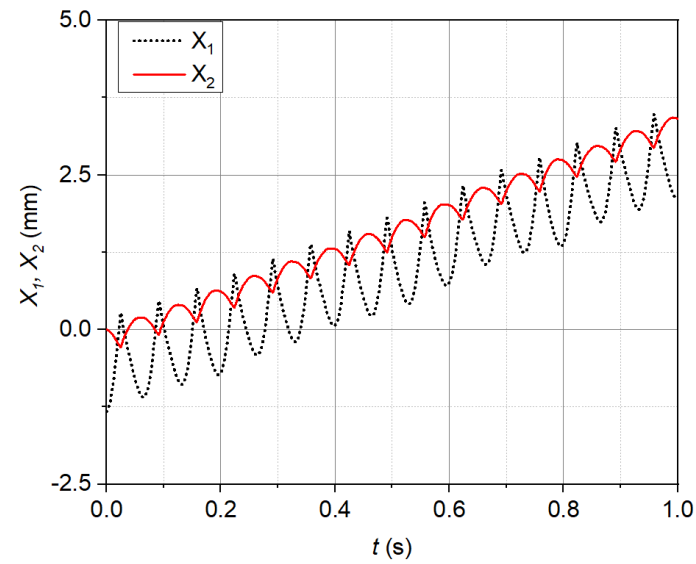

(a)

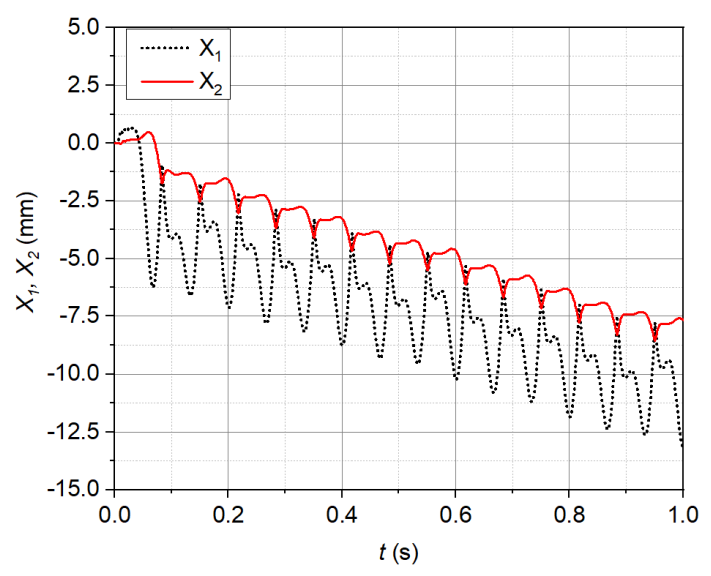

(c)

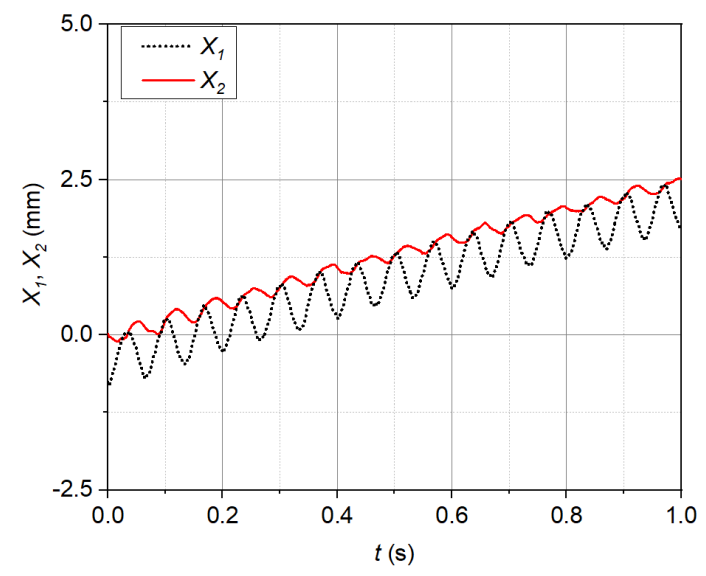

(b)

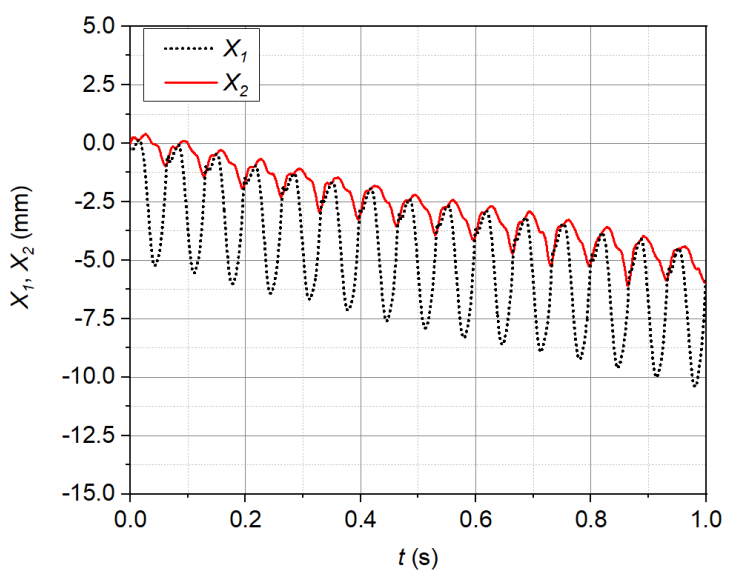

(d)

Fig. 5. Time histories of the displacement, $X_{1}$ of the mass $m_{1}$ and $X_{2}$ of the system body obtained numerically $(\mathrm{a}, \mathrm{c})$ and experimentally $(\mathrm{b}, \mathrm{d})$. A set of parameters: $f=15 \mathrm{~Hz}, \alpha=1.19$ were applied. The friction force of $2.3 \mathrm{~N}$ was applied in sub-plots $(\mathrm{a}, \mathrm{b})$ and $13.6 \mathrm{~N}$ in sub-plots $(\mathrm{c}, \mathrm{d})$ 
it can be seen that with the same force ratio $\alpha=1.19$, the system has the same backward motion under high friction $\left(F_{S}=13.6 \mathrm{~N}\right.$, as shown in Figs. $5(\mathrm{c})$ and $\left.5(\mathrm{~d})\right)$ in both simulation and experiments. Besides, the forward motion was found under low friction $\left(F_{s}=2.3 \mathrm{~N}\right.$, as depicted in Figs. 5(a) and 5(b)).

Being experimentally validated, the mathematical model now can be used to scrutinize the system response for different values of parameters.

\section{RESPONSE COMPARISON}

In this section, the two models, i.e. the vibro-impact driven and pure-vibration driven systems, will be compared. Two criteria will be used in comparative study: the progression rate (including the magnitude and direction of the motion obtained) and the dynamic response.

\subsection{Non-dimensional models}

In order to compare and analysis the two systems, the two models were transformed to dimensionless forms, by using the following terms

$$
\begin{aligned}
& \tau=\Omega_{0} t ; x_{1}=\frac{k_{1}}{F_{b}} X_{1} ; x_{2}=\frac{k_{1}}{F_{b}} X_{2} ; \Omega_{0}=\sqrt{\frac{k_{1}}{m_{1}}} ; \omega=\frac{\Omega}{\Omega_{0}} ; \\
& \zeta=\frac{c}{2 m_{1} \Omega_{0}} ; \chi=\frac{A}{F_{b}} ; \sigma=\frac{k_{0}}{k_{1}} ; \beta=\frac{k_{2}}{k_{1}}\left(\frac{F_{b}}{k_{1}}\right)^{2} ; \gamma=\frac{k_{1}}{F_{b}} G ; \mu=\frac{m_{2}}{m_{1}} ; f_{r}=\frac{F_{r}}{F_{b}}
\end{aligned}
$$

where $F_{b}$ is a force reference. Here we introduce a new force ratio, $\chi$ instead of the force ratio $\alpha$ in Eq. (3). Using the new force ratio, the excitation force variation can be independently examined at different levels of the friction force.

For pure-vibration driven by half-sine excitation force, the non-dimension model now is expressed as

$$
\left\{\begin{array}{l}
x_{1}^{\prime}=v_{1} \\
v_{1}^{\prime}=\chi \sin (\omega \tau) \cdot h_{v}-\left(x_{1}-x_{2}\right)-\beta\left(x_{1}-x_{2}\right)^{3}-2 \zeta\left(\frac{d x_{1}}{d \tau}-\frac{d x_{2}}{d \tau}\right) \\
x_{2}^{\prime}=v_{2} \\
v_{2}^{\prime}=\left[-\chi \sin (\omega \tau) \cdot h_{v}+\left(x_{1}-x_{2}\right)+\beta\left(x_{1}-x_{2}\right)^{3}+2 \zeta\left(\frac{d x_{1}}{d \tau}-\frac{d x_{2}}{d \tau}\right)-f_{r} \operatorname{sgn}\left(\frac{d x_{2}}{d \tau}\right)\right] \frac{1}{\mu}
\end{array}\right.
$$

where

$$
\begin{cases}h_{v}=1, & \sin (\omega \tau)>0 \\ h_{v}=0, & \text { else }\end{cases}
$$


The dimensionless form of the vibro-impact driven system (Eq. (4)) is now described as following

$$
\left\{\begin{array}{l}
x_{1}^{\prime}=v_{1} \\
v_{1}^{\prime}=\chi \sin (\omega \tau)-\left(x_{1}-x_{2}\right)-\beta\left(x_{1}-x_{2}\right)^{3}-2 \zeta\left(\frac{d x_{1}}{d \tau}-\frac{d x_{2}}{d \tau}\right)-h \sigma\left(x_{1}-x_{2}-\gamma\right) \\
x_{2}^{\prime}=v_{2} \\
v_{2}^{\prime}=\left[-\chi \sin (\omega \tau)+\left(x_{1}-x_{2}\right)+\beta\left(x_{1}-x_{2}\right)^{3}+2 \zeta\left(\frac{d x_{1}}{d \tau}-\frac{d x_{2}}{d \tau}\right)+h \sigma\left(x_{1}-x_{2}-\gamma\right)-f_{r} \operatorname{sgn}\left(\frac{d x_{2}}{d \tau}\right)\right] \frac{1}{\mu}
\end{array}\right.
$$

where $h$ is the Heaviside function describing the impact conditions in the dimensionless form, i.e.

$$
\begin{cases}h=1, & x_{1}-x_{2}-\gamma>0 \\ h=0, & x_{1}-x_{2}-\gamma \leq 0\end{cases}
$$

The two models described by Eq. (12) and Eq. (14) will be used to compare the two systems, pure-vibration and vibro-impact driven locomotion systems. The solutions were obtained with $\chi \in[0,2] ; f_{r} \in[0,2] ; \beta=3.113 ; \zeta=0.119 ; \sigma=657.74 ; \gamma=0.047$ and $\mu=3.586$.

\subsection{Comparison of progression rate}

The progression rate $P$ of each system was defined as the displacement of the system, $x_{2}$ obtained at $\tau=100$. The results for each model were obtained using the same set of all input parameters. Fig. 6 presents two surface plots of the progression rate of the two systems with respects to two parameters: the friction $f_{r}$ and the excitation force $\chi$.

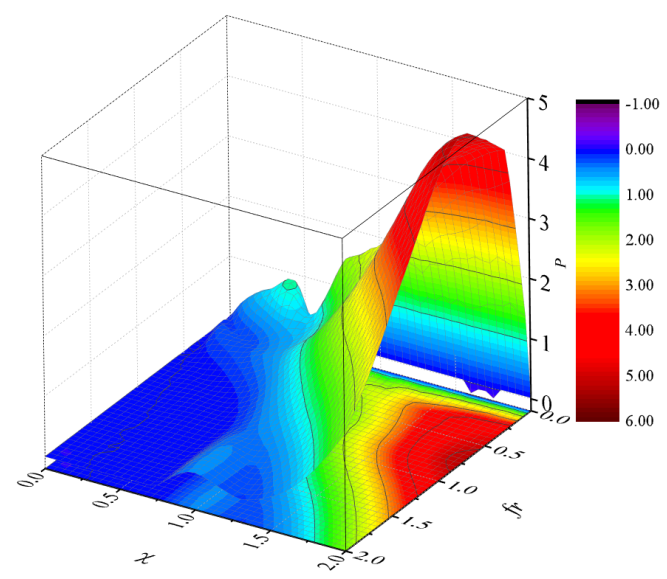

(a) Pure-vibration

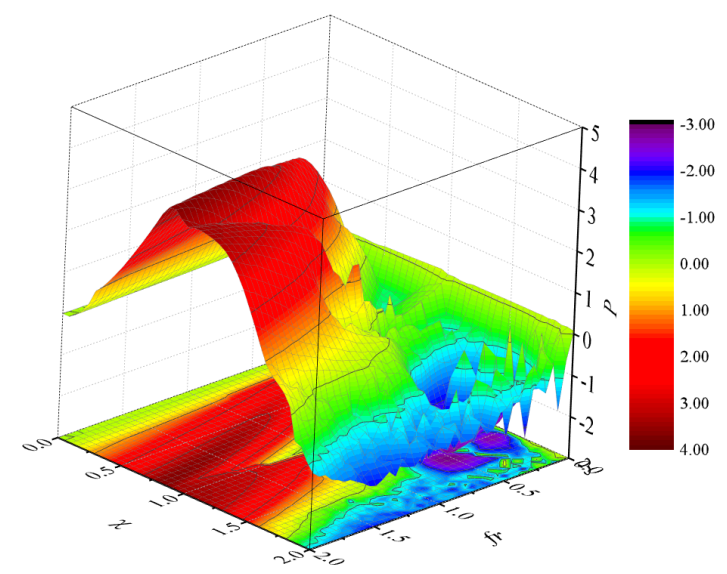

(b) Vibro-impact

Fig. 6. (Color online) Surface plots of the progression rate with respect to the friction and the excitation levels

As can be seen in Fig. 6, the two systems obtained different progression rate, both forward $(P>0)$ and backward $(P<0)$ for different values of the friction and excitation 
forces. Regarding the highest progression, the pure-vibration system provided a larger distance (more than 4) compared to the vibro-impact system (less than 4). Fig. 7 shows the contour plot of the progression rates, presenting a clearer view of the differences between the two systems.

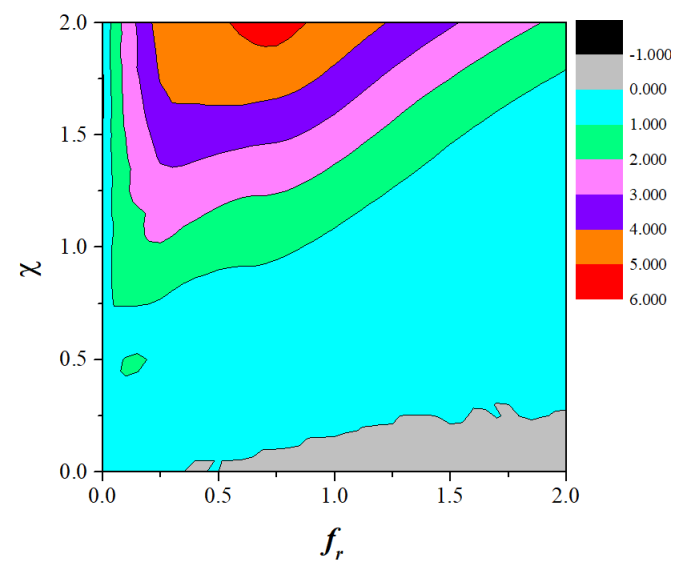

(a) Pure-vibration

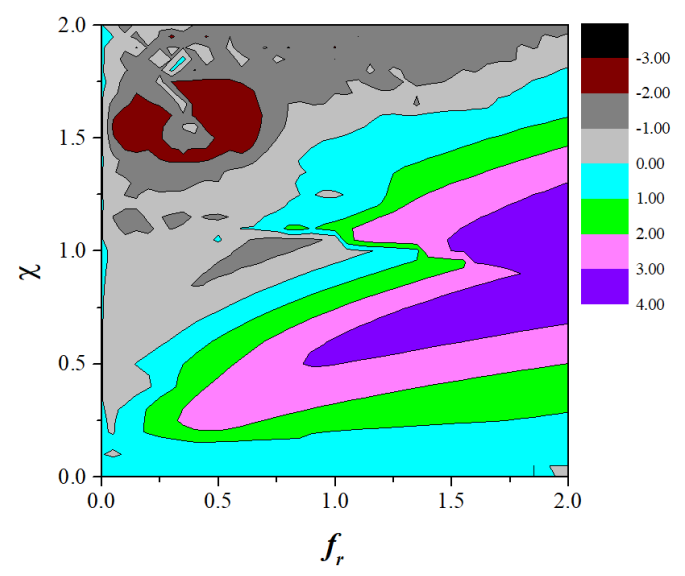

(b) Vibro-impact

Fig. 7. (Color online) Contour plots of the progression rate with respect to the friction and the excitation levels

In Fig. 7, the areas of backward motion were represented by gray, dark grey and wine, whereas the areas of forward motion were shown in cyan, green, magenta, violet, orange, and red. As can be seen, the pure-vibration driven system provided forward motion for almost values of friction and excitation forces. There was a small range of $f_{r} \in[0.5,2]$ combined with $\chi<0.25$ resulted in backward motion. In contrast, the vibroimpact provided both backward and forward motions with around 50 percent for each case. The backward motion appeared at high values of the excitation $\chi$ combined with almost all values of friction $f_{r}$. It can be remarked that, if a forward motion under low friction condition is expected, the pure-vibration with large excitation is recommended. In order to save energy consumption, the vibro-impact model with small excitation force should be applied.

\subsection{Comparison of dynamics response}

The dynamics response of the system was firstly examined by using bifurcation analysis. In this stage, the friction force, $f_{r}$ was considered as a bifurcation parameter. The bifurcation diagram represents the velocity map, $V^{*}$, defined as a projection of the Poincare map on the velocity axis of the phase plane. The plotting process is generated as below. For each value of the friction, $f_{r}$, the relative velocity maps, $V^{*}$ were calculated. The progress were run for 300 cycles of the excitation force. The first 100 cycles were omitted as transient situation. The next 200 points of the steady state of the velocity map was used to plot the bifurcation. 
Fig. 8 shows the bifurcation diagram of the two systems with the same input parameters. As can be seen, in this case, both systems have a likely period-one motion. The bifurcation curve appeared as a single line for pure-vibration, but in a slightly fluctuated curve for vibro-impact system.

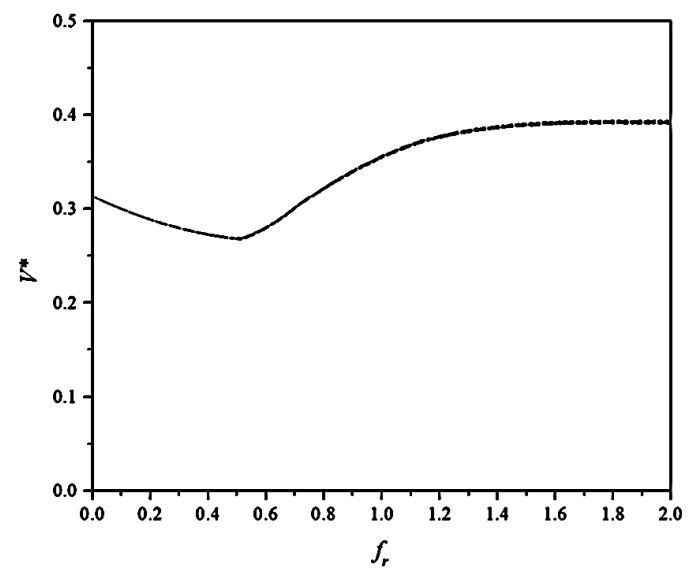

(a) Pure-vibration

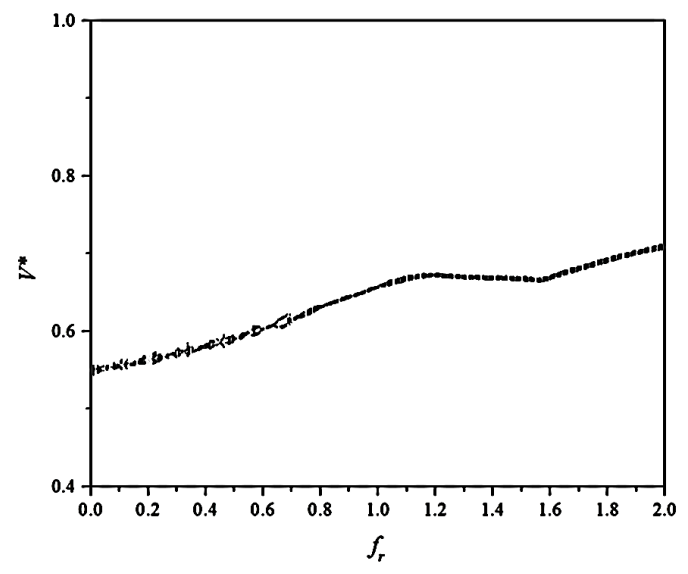

(b) Vibro-impact

Fig. 8. Bifurcation diagram of the pure-vibration (a) and vibro-impact (b) for friction force, $f_{r} \in[0,2]$; A set of $\chi=1 ; \omega=1$ was applied

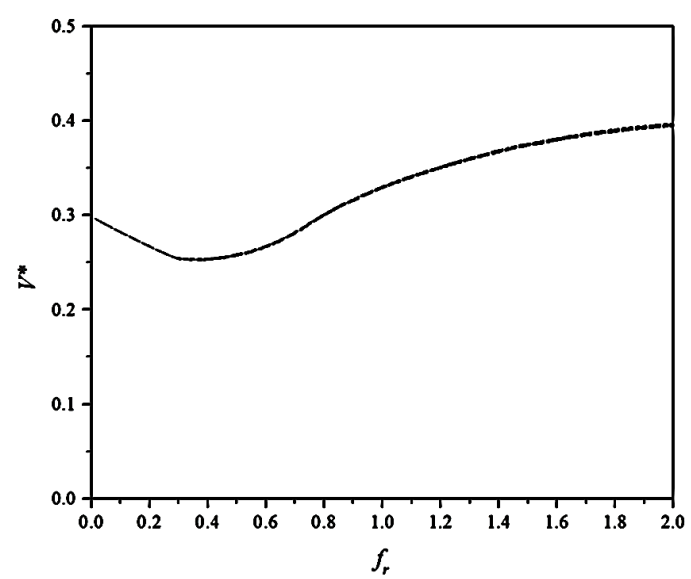

(a) Pure-vibration

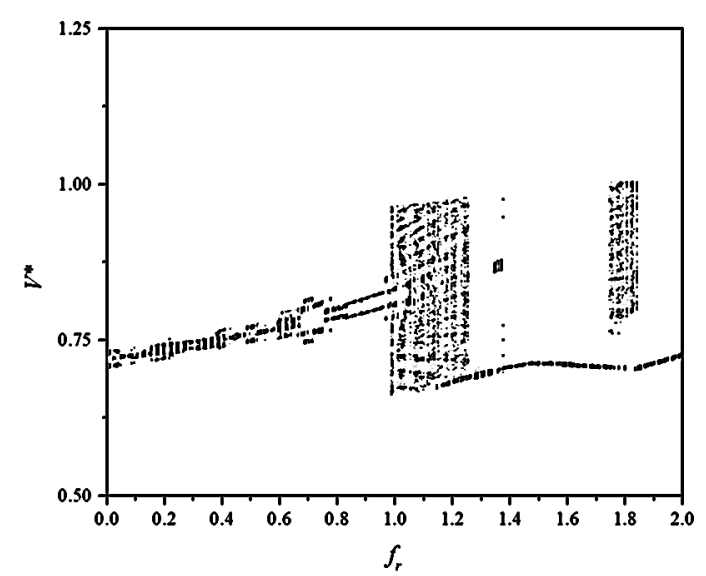

(b) Vibro-impact

Fig. 9. Bifurcation diagram of the pure-vibration (a) and vibro-impact (b) for friction force,

$$
f_{r} \in[0,2] \text {; A set of } \chi=1.5 ; \omega=1 \text { was applied }
$$

For the excitation force larger than 1, Fig. 9 shows the bifurcation diagrams of the two systems with $\chi=1.5, \omega=1$, and $f_{r} \in[0,2]$. As can be seen in Fig. 9(a), the purevibration always has period-1 motion. In case of the vibro-impact system (Fig. 9(b)), for 
lower range of the friction force, $f_{r} \in[0,1]$, the bifurcation appeared in narrow bands, as can be considered possibly a motion of period- 1 and period-2. For a larger range, $f_{r} \in[1,2]$, the system responses in both chaotic motion in some sub-ranges and period-1 in the other sub-ranges. Dynamics response of the two systems will be compared using the basin of attraction analysis.

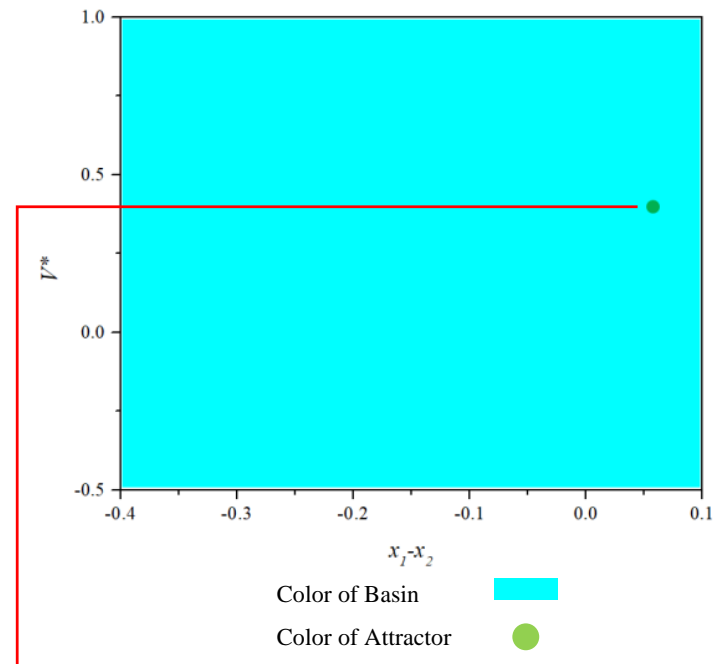

(a) Basin of attraction of pure-vibration

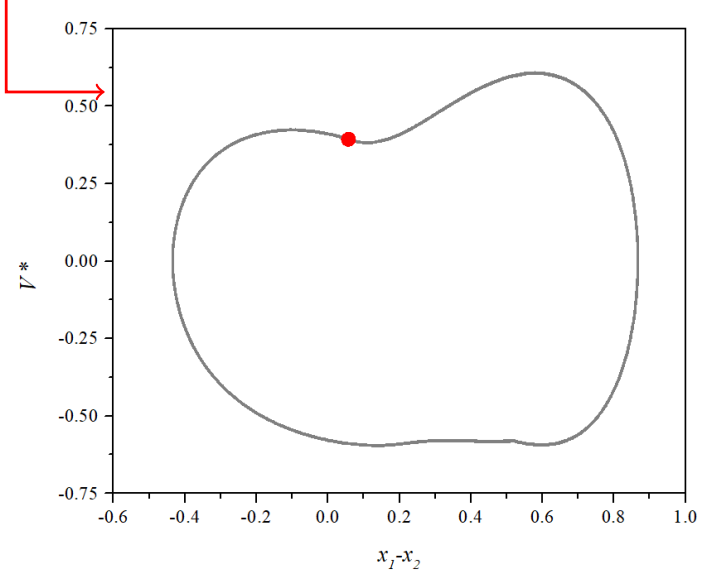

(c) Phase portrait (grey lines) and Poincaré maps (red dots) of pure-vibration

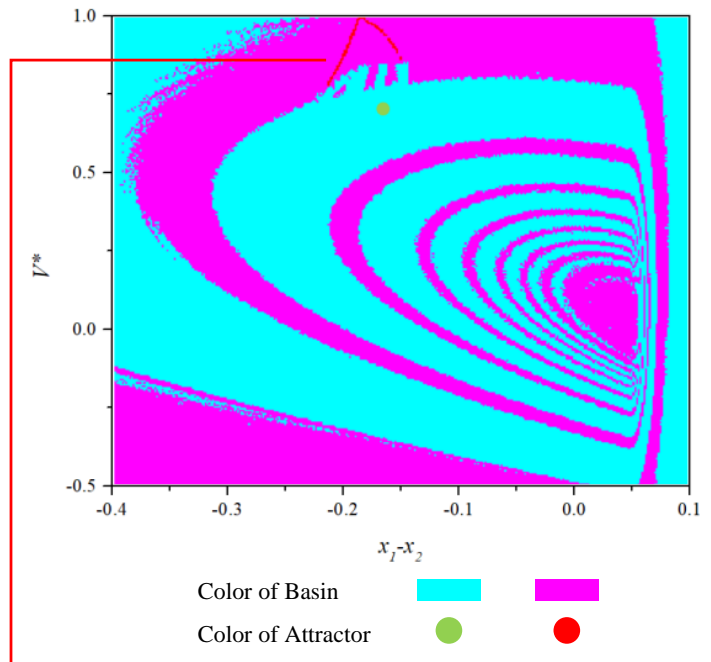

(b) Basin of attraction of vibro-impact

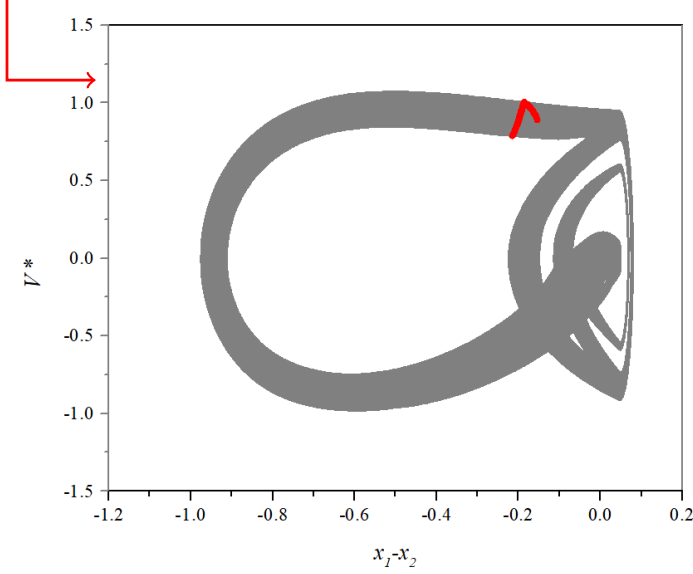

(d) Phase portrait (grey lines) and Poincaré maps (red dots) of vibro-impact

Fig. 10. Basin of attraction and phase portrait combined with Poincaré maps of the two systems; the results obtained for calculated for $\chi=1.5 ; \omega=1 ; f_{r}=1.8$

Fig. 10 shows basin of attraction plots and a phase portrait combined with the Poincare section as illustrations. For basin of attractions, a set of initial conditions of -0.4 to 0.1 for 
the relative displacement and -0.5 to 1 for the relative velocity was remained for both two cases of pure-vibration and vibro-impact systems.

For the case of pure-vibration (Fig. 10(a)), the attractor appeared in a small green dot, reflecting that the system has period-1 motion. The Poincare maps (Fig. 10(c)) appeared in only one red dot confirmed this behavior. For vibro-impact system (see Fig. 10(b)), the attractor appeared to be chaotic. In this figure, the chaotic attractor is described by red color, and its basin was shown in magenta. A co-existing attractor of period-1 motion shown in a green dot, with the basin in cyan. Fig. 10(d) presents the phase plot and Poincaré map corresponding to the chaotic attractor. The Poincaré map distributed in a zigzag curve reflected the chaotic response of the system.

Looking at the basin of attraction and Poinacare map, it can be confirmed that the friction force has substantial effects on the dynamic response of the two systems at different ways. The pure-vibration always has period-1 motion whereas the vibro-impact can be chaotic for several values of the friction force.

\section{CONCLUSIONS}

This paper presented comparison results of two locomotion systems: one is purevibration driven system and another is a vibro-impact device. The experimental setup allows examine the friction force as a variable while keeping the weight of the whole system as constant. The mathematical models of the two systems were experimentally verified. The dimensionless form of the two system was developed, allowing to expand the results to other sizes of the system in practice. Bifurcation analysis and basin of attraction were applied to scrutinize the system response with respects to the friction. The significant remarks would be noted as follow:

- To achieve forward motion, the pure-vibration is recommended to work under small friction condition, using high excitation force. In contrast, the vibro-impact can provide forward motion with small excitation force. The highest progression rate of the vibro-impact was less than that of the pure-vibration system in the investigated ranges of input parameters.

- The pure-vibration always has period-1 motion, whereas the vibro-impact system has abundant dynamic response of the system, from period-1, period-2 to chaotic motions.

Further studies would be made to provide more beneficial recommendation in design and operation of the self-propelled locomotion systems.

\section{ACKNOWLEDGEMENT}

This research is funded by Vietnam National Foundation for Science and Technology Development (NAFOSTED) under grant number 107.01-2017.318.

\section{REFERENCES}

[1] A. Tharakeshwar and A. Ghosal. A three-wheeled mobile robot for traversing uneven terrain without slip: Simulation and experiments. Mechanics Based Design of Structures and Machines, 41, (1), (2013), pp. 60-78. https://doi.org/10.1080/15397734.2012.703606. 
[2] B. He, B. R. Wang, T. H. Yan, and Y. Y. Han. A distributed parallel motion control for the multi-thruster autonomous underwater vehicle. Mechanics Based Design of Structures and Machines, 41, (2), (2013), pp. 236-257. https://doi.org/10.1080/15397734.2012.726847.

[3] J. Cazalilla, M. Vallés, Á. Valera, V. Mata, and M. Díaz-Rodríguez. Hybrid force/position control for a 3-DOF 1T2R parallel robot: Implementation, simulations and experiments. Mechanics Based Design of Structures and Machines, 44, (1-2), (2016), pp. 16-31. https://doi.org/10.1080/15397734.2015.1030679.

[4] A. Shukla and H. Karki. Application of robotics in onshore oil and gas industry-A review Part I. Robotics and Autonomous Systems, 75, (2016), pp. 490-507. https://doi.org/10.1016/j.robot.2015.09.012.

[5] A. Garg, C. S. Vikram, S. Gupta, M. K. Sutar, P. M. Pathak, N. K. Mehta, A. K. Sharma, and V. K. Gupta. Design and development of in vivo robot for biopsy. Mechanics Based Design of Structures and Machines, 42, (3), (2014), pp. 278-295. https://doi.org/10.1080/15397734.2014.898587.

[6] F. Chernous'ko. The optimum rectilinear motion of a two-mass system. Journal of Applied Mathematics and Mechanics, 66, (1), (2002), pp. 1-7. https://doi.org/10.1016/s00218928(02)00002-3.

[7] A. Nunuparov, F. Becker, N. Bolotnik, I. Zeidis, and K. Zimmermann. Dynamics and motion control of a capsule robot with an opposing spring. Archive of Applied Mechanics, 89, (10), (2019), pp. 2193-2208. https://doi.org/10.1007/s00419-019-01571-8.

[8] M. N. Huda and H. Yu. Trajectory tracking control of an underactuated capsubot. $A u$ tonomous Robots, 39, (2), (2015), pp. 183-198. https://doi.org/10.1007/s10514-015-9434-3.

[9] G. Su, C. Zhang, R. Tan, and H. Li. A design of the electromagnetic driver for the internal force-static friction capsubot. In 2009 IEEE/RSJ International Conference on Intelligent Robots and Systems, IEEE, (2009), https:/ / doi.org/10.1109/IROS.2009.5354587.

[10] H. Li, K. Furuta, and F. L. Chernousko. Motion generation of the capsubot using internal force and static friction. In Proceedings of the 45th IEEE Conference on Decision and Control, IEEE, (2006), https: / / doi.org/10.1109/CDC.2006.377472.

[11] P. Liu, M. N. Huda, Z. Tang, and L. Sun. A self-propelled robotic system with a visco-elastic joint: dynamics and motion analysis. Engineering with Computers, 36, (2019), pp. 655-669. https://doi.org/10.1007/s00366-019-00722-3.

[12] N. A. Sobolev and K. S. Sorokin. Experimental investigation of a model of a vibration-driven robot with rotating masses. Journal of Computer and Systems Sciences International, 46, (2007), pp. 826-835. https://doi.org/10.1134/s1064230707050140.

[13] H. Yu, Y. Liu, and T. Yang. Closed-loop tracking control of a pendulum-driven cart-pole underactuated system. Proceedings of the Institution of Mechanical Engineers, Part I: Journal of Systems and Control Engineering, 222, (2008), pp. 109-125. https://doi.org/10.1243/09596518jsce460.

[14] V.-D. Nguyen and N.-T. La. An improvement of vibration-driven locomotion module for capsule robots. Mechanics Based Design of Structures and Machines, (2020), pp. 1-15. https://doi.org/10.1080/15397734.2020.1760880.

[15] E. Pavlovskaia, M. Wiercigroch, and C. Grebogi. Modeling of an impact system with a drift. Physical Review E, 64, (2001). https://doi.org/10.1103/physreve.64.056224.

[16] V.-D. Nguyen, K.-C. Woo, and E. Pavlovskaia. Experimental study and mathematical modelling of a new of vibro-impact moling device. International Journal of Non-Linear Mechanics, 43, (2008), pp. 542-550. https://doi.org/10.1016/j.ijnonlinmec.2007.10.003. 
[17] V. D. Nguyen and K. C. Woo. New electro-vibroimpact system. Proceedings of the Institution of Mechanical Engineers, Part C: Journal of Mechanical Engineering Science, 222, (2008), pp. 629-642. https://doi.org/10.1243/09544062jmes833.

[18] A. N. Grankin and S. F. Yatsun. Investigation of vibroimpact regimes of motion of a mobile microrobot with electromagnetic drive. Journal of Computer and Systems Sciences International, 48, (2009), pp. 155-163. https://doi.org/10.1134/s1064230709010158.

[19] K. A. Sapronov, A. A. Cherepanov, and S. F. Yatsun. Investigation of motion of a mobile two-mass vibration-driven system. Journal of Computer and Systems Sciences International, 49, (2010), pp. 144-151. https://doi.org/10.1134/s1064230710010156.

[20] Y. Liu, E. Pavlovskaia, and M. Wiercigroch. Experimental verification of the vibro-impact capsule model. Nonlinear Dynamics, 83, (2015), pp. 1029-1041. https://doi.org/10.1007/s11071-015-2385-6.

[21] V.-D. Nguyen, T.-H. Duong, N.-H. Chu, and Q.-H. Ngo. The effect of inertial mass and excitation frequency on a Duffing vibro-impact drifting system. International Journal of Mechanical Sciences, 124-125, (2017), pp. 9-21. https://doi.org/10.1016/j.ijmecsci.2017.02.023.

[22] V.-D. Nguyen, H.-D. Ho, T.-H. Duong, N.-H. Chu, and Q.-H. Ngo. Identification of the effective control parameter to enhance the progression rate of vibro-impact devices with drift. Journal of Vibration and Acoustics, 140, (2017). https://doi.org/10.1115/1.4037214.

[23] Y. Liu, E. Pavlovskaia, M. Wiercigroch, and Z. Peng. Forward and backward motion control of a vibro-impact capsule system. International Journal of Non-Linear Mechanics, 70, (2015), pp. 30-46. https://doi.org/10.1016/j.ijnonlinmec.2014.10.009.

[24] T.-H. Duong, V.-D. Nguyen, H.-C. Nguyen, N.-P. Vu, N.-K. Ngo, and V.-T. Nguyen. A new design for bidirectional autogenous mobile systems with two-side drifting impact oscillator. International Journal of Mechanical Sciences, 140, (2018), pp. 325-338. https://doi.org/10.1016/j.ijmecsci.2018.01.003.

[25] A. Ivanov. Analysis of an impact-driven capsule robot. International Journal of Non-Linear Mechanics, 119, (2020). https://doi.org/10.1016/j.ijnonlinmec.2019.103257.

[26] J. Fan, C. Li, Z. Yang, S. Chen, J. Cao, and C. Dou. On discontinuous dynamics of a 2-DOF oscillator with an one-sided rigid obstacle. International Journal of Non-Linear Mechanics, 118, (2020). https://doi.org/10.1016/j.ijnonlinmec.2019.103261.

[27] Y. Liu, M. Wiercigroch, E. Pavlovskaia, and H. Yu. Modelling of a vibro-impact capsule system. International Journal of Mechanical Sciences, 66, (2013), pp. 2-11. https://doi.org/10.1016/j.ijmecsci.2012.09.012.

[28] X. D. Gu and Z. C. Deng. Dynamical analysis of vibro-impact capsule system with Hertzian contact model and random perturbation excitations. Nonlinear Dynamics, 92, (2018), pp. 17811789. https://doi.org/10.1007/s11071-018-4161-x.

[29] Y. Liu, S. Islam, E. Pavlovskaia, and M. Wiercigroch. Optimization of the vibro-impact capsule system. Strojniški vestnik - Journal of Mechanical Engineering, 62, (2016), pp. 430-439. https://doi.org/10.5545/sv-jme.2016.3754. 


\section{NOMENCLATURE}

A Amplitude of the excitation force (N)

c Damping coefficient (Ns/m)

$f \quad$ Frequency $(\mathrm{Hz})$

$F_{r} \quad$ Friction force $(\mathrm{N})$

$f_{r} \quad$ Dimensionless friction force

$F_{\text {spr }} \quad$ Spring force (N)

$H$ Heaviside function

$k_{1} \quad$ Linear spring stiffness $(\mathrm{N} / \mathrm{m})$

$k_{2} \quad$ Cubic spring stiffness $\left(\mathrm{N} / \mathrm{m}^{3}\right)$

$X_{1} \quad$ Absolute displacement of the inside mass (m)

$x_{1} \quad$ Dimensionless displacement of the inside mass

$X_{2} \quad$ Absolute displacement of the body mass ( $m$ )

$x_{2} \quad$ Dimensionless displacement of the body mass $\alpha \quad$ Force ratio

$\beta$ Dimensionless cubic stiffness

$\chi \quad$ New force ratio

$\gamma$ Dimensionless gap

$\eta \quad$ Dimensionless Heaviside function

$\mu$ Mass ratio

$\sigma \quad$ Dimensionless impact stiffness

$\tau$ Time (s)

$\tau$ Dimensionless time

$\Omega \quad$ Angular frequency of the excitation force ( $\mathrm{rad} / \mathrm{s})$

$\Omega \quad$ Natural angular frequency of the system ( $\mathrm{rad} / \mathrm{s})$

$\omega$ Dimensionless angular frequency of the excitation force

$\zeta$ Dimensionless damping coefficient 